\title{
Modélisation des flux sédimentaires charriés dans la baie du Mont-Saint-Michel (Manche, France)
}

\section{Bedload transport modelisation in a bay characterized by a macrotidal environment: example of the Mont-Saint-Michel Bay (Manche, France)}

\author{
A. Ehrhold ${ }^{\text {a, } *, \text { S. Guillou }}{ }^{\text {b }}$, J.P. Auffret ${ }^{\text {c }}$, T. Garlan ${ }^{\text {a }}$, K. Dan Nguyen ${ }^{\text {d }}$ \\ a Ifremer DEL Brest, Ifremer technopole de Brest-Iroise, BP 70, 29280 Plouzané, France \\ ${ }^{b}$ Lusac, site universitaire de Cherbourg, BP 78, 50130 Octeville, France \\ ${ }^{c}$ Unité morpholodynamique continentale et côtière, ER 109 université de Caen, 14032 Caen cedex, France \\ ${ }^{d}$ Département de mathématiques et mécanique, boulevard Maréchal-Juin, université de Caen Campus II, BP 5186, Caen cedex, France \\ Reçu le 8 février 2003 ; révisé et accepté le 27 février 2003
}

\section{Résumé}

Cette étude des flux sédimentaires en baie du Mont-Saint-Michel combine l'utilisation d'un modèle numérique 2DH résolvant les équations de Saint-Venant et un modèle des transits sédimentaires établis sur l'analyse des levés d'imagerie acoustique calibrée par un canevas dense de prélèvements sédimentaires. Les simulations des flux sédimentaires aux abords du Mont-Saint-Michel montrent le cheminement des sables coquilliers depuis les fonds subtidaux au large de la côte de Saint-Malo jusqu'aux encablures du Mont-Saint-Michel à l'est et la région de Cancale à l'ouest. Ce transfert des sédiments à la côte est à l'origine de la construction du prisme holocène dans cette région. Le modèle physique reproduit la dynamique tidale des sédiments aux abords immédiats du Mont-Saint-Michel sous le jeu de l'asymétrie des courants de marée et montre l'intérêt du couplage modèle numérique-dynamique de terrain dans la compréhension des processus de comblement des baies de la façade Manche-Atlantique.

(C) 2003 Éditions scientifiques et médicales Elsevier SAS and Ifremer/CNRS/IRD. Tous droits réservés.

\begin{abstract}
A study of the sediment bedload in the Mont-Saint-Michel bay is presented here. It combines the use of a $2 \mathrm{DH}$ numerical model and a pattern of sediment bedload transport established on the analysis of the statements of sidescan imagery combined with a dense groundwork of sedimentary grab samples. Results show the role of tidal currents asymetry in the realistic bioclastic sand transfert from the subtidal environment to the coast and the divergence of sedimentary dynamic between the maritime bay to the West and the tidal delta to the East. This in-shore transfer is responsable of the Holocene prism construction. The model reproduces tidal sedimentary dynamics with the immediate surroundings of the Mont-Saint-Michel. It shows the interest of the modeling of bedload flows in the reconstitution of the sedimentary processes in the filling bays of Manche-Atlantic coast.
\end{abstract}

(C) 2003 Éditions scientifiques et médicales Elsevier SAS and Ifremer/CNRS/IRD. Tous droits réservés.

Mots clés : Modèle hydrodynamique ; Débit massique ; Sonar latéral ; Sédiment ; Baie du Mont-Saint-Michel

Keywords: Hydrodynamic model; Bedload transport; Sidescan; Sediment; Mont-Saint-Michel bay

* Auteur correspondant.

Adresse e-mail : aehrhold@ifremer.fr (A. Ehrhold). 


\section{Introduction}

La baie du Mont-Saint-Michel constitue un bassin sédimentaire côtier dominé par la marée sur une surface d'environ $600 \mathrm{~km}^{2}$ Fig. 1. Les profondeurs y sont modestes, inférieures à $20 \mathrm{~m}$, et les conditions de marnages exceptionnelles, atteignent $14 \mathrm{~m}$ en vive-eau (VE) d'équinoxe. Alors que le littoral est par ailleurs en recul, on assiste dans la baie à un exhaussement des fonds, particulièrement marqué depuis un demi-siècle dans la région estuarienne. Ce comblement, qui se traduit par une avancée spectaculaire du schorre (Larsonneur, 1989), est nuisible au caractère maritime du site. L'objet de cette étude est la détermination des flux de sédiments non-cohésifs dans les processus de comblement de cette baie.

Les modes et les voies de transport à l'origine de ce comblement étaient mal connus sans l'utilisation de l'imagerie acoustique par sonar à balayage latéral basée sur le principe de rétrodiffusion des sédiments (Berné, 1991). Pour identifier qualitativement les voies de transport des sédiments en charriage du large vers la côte, plusieurs campagnes au sonar à balayage latéral modèle EGG 272 TH ont été réalisées entre 1995 et 1997 dans tout le secteur de la baie du Mont-Saint-Michel jusqu'aux îles Chausey (Ehrhold, 1999). Jusqu'à présent, l'ensemble des études de dynamique sédimentaire réalisées dans cette baie concernait essentiellement

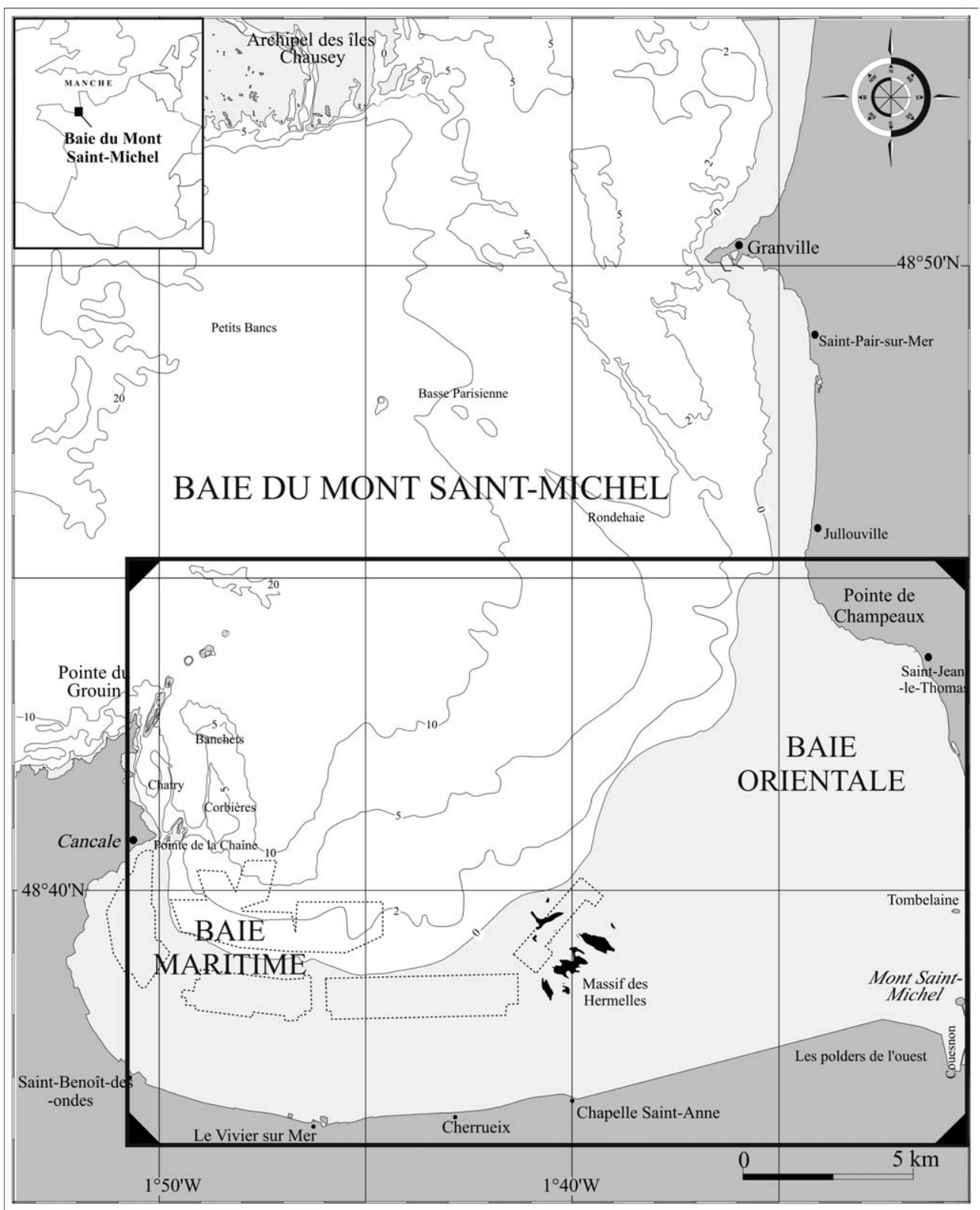

Fig. 1. Carte générale de la baie du Mont-Saint-Michel et du secteur d'étude. General map of the Mont-Saint-Michel bay and the study area. 


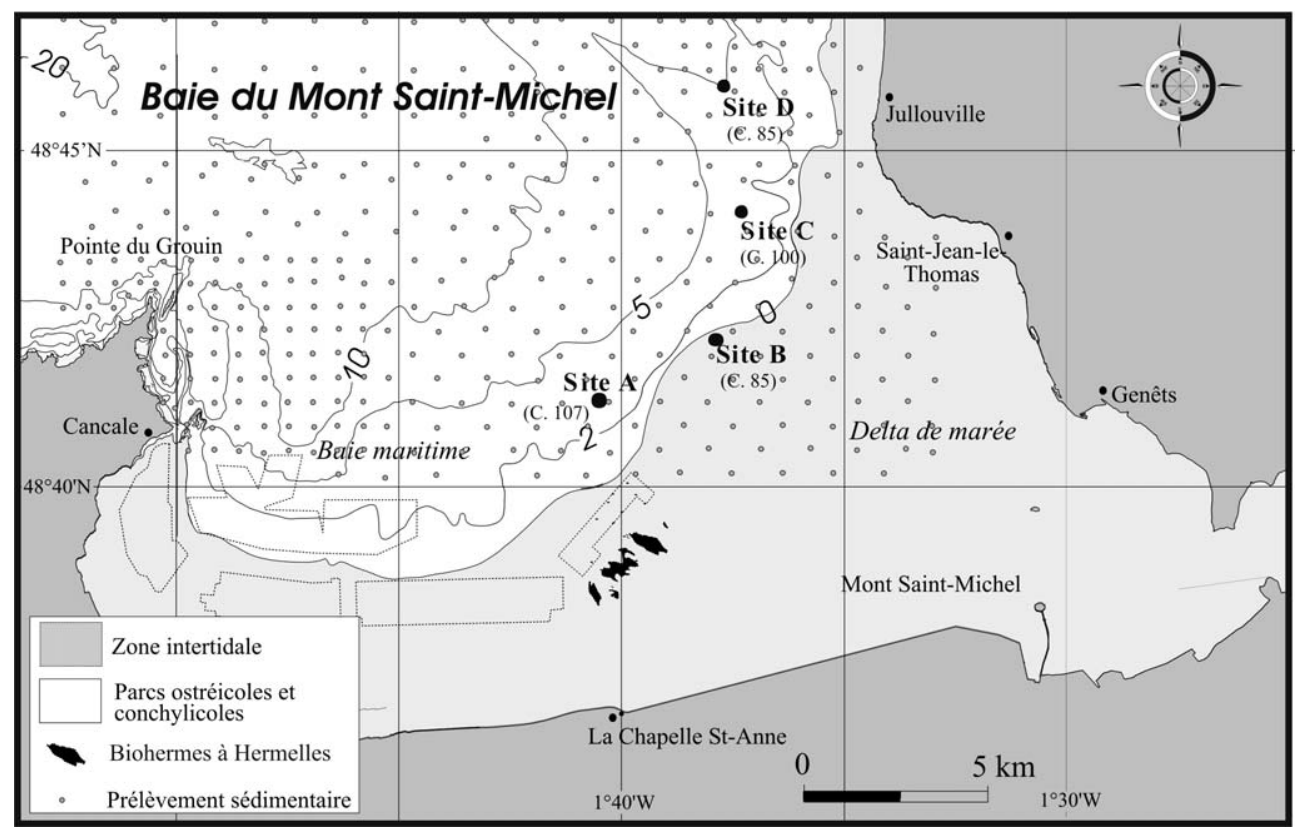

Fig. 2. Morphologie de la Baie du Mont-Saint-Michel (A, B, C, D ; mesures des courants sur le fond, C.100 : coefficient de marée). Morphology of the Mont-Saint-Michel bay (A, B, C, D; near-bottom current measurements, C.100: coefficient of the tide).

l'estran (Nikodic, 1981 ; Compain et al., 1988 ; Larsonneur, 1989 ; Bonnot-Courtois, 1994 ; Levoy, 1994). Seul Walker (2001) avait réalisé entre 1982 et 1986 quelques profils avec un sonar similaire dans cette partie sud du golfe normandbreton.

Dans cette étude l'insonification des fonds sur une distance cumulée de $830 \mathrm{~km}$ et une fauchée de $300 \mathrm{~m}$ a fourni un schéma précis de la répartition des figures sédimentaires construites par les courants de marée. L'étude morphologique de ces corps sédimentaires donne des informations précises sur les directions et les sens de transports sédimentaires (Kenyon, 1970 ; Berné, 1991 ; Thauront, 1994). L’asymétrie des grandes dunes hydrauliques modelant le prisme sableux au large de Granville ou de Cancale et les figures sédimentaires longitudinales (rubans sableux, sillon d'érosion, queue de comète, traînées sableuses) sont des indicateurs de la direction du transport sédimentaire résiduel sur le fond (Belderson et al., 1972). Les petites et moyennes dunes hydrauliques (amplitude inférieure à $50 \mathrm{~cm}$ ) n'ont pas été prises en compte pour établir le schéma général des directions de transport sédimentaire dans cette partie du golfe normandbreton en raison de leur aptitude à s'inverser sous l'effet d'un régime de courant alternatif important (Allen, 1984 ; Walker, 2001). À chaque fois qu'il l'a été possible, la direction et le sens des transports ont été reportés sous la forme d'un vecteur en chacun des points de prélèvement.

Depuis la pointe du Grouin jusqu'à Jullouville, une large zone se découvre à marée basse pour une vive-eau d'équinoxe représentant une surface de $220 \mathrm{~km}^{2}$ et rendant très difficile la prospection avec un sonar latéral remorqué derrière un navire océanographique côtier. Dans ces conditions, la détermination des flux de sédiments non-cohésifs par les modèles numériques devient complémentaire lorsque les données objectives de terrain sont insuffisantes. Orbi (1986), Larsonneur et Walker (1986) et Grochoswski et al. (1993) ont utilisé un modèle numérique pour prédire les débits solides résiduels à l'échelle, respectivement, du golfe normandbreton et de la Manche. Nous proposons d'utiliser ici un modèle traitant les zones subtidales et découvrantes, en association avec les résultats de l'interprétation des sonogrammes et des analyses granulométriques sur les prélèvements effectués dans la zone d'étude (Ehrhold, 1999).

\section{Les données hydrosédimentaires}

\subsection{Mesures ponctuelles in situ}

Les sédiments des fonds marins de la zone d'étude entre l'archipel des îles Chausey et le Mont-Saint-Michel ont été prélevés à partir d'une benne Shipeck et Smith pour les sédiments grossiers du large à caractère graveleux. Plus de 600 prélèvements sédimentaires localisés au GPS différentiel ont ainsi été récoltés entre janvier 1995 et août 1997. La distance entre les points varie de 900 à $2000 \mathrm{~m}$ Fig. 2]. Les analyses granulométriques ont été réalisées sur le sédiment total non décarbonaté et les paramètres sédimentologiques obtenus par la méthode des moments statistiques (SeawardThomson et Hails, 1973).

Quatre courantomètres électromagnétiques de type Interocean S4 (fréquence d'acquisition de $2 \mathrm{~Hz}$ ) ont été mouillés durant une marée par temps calme (agitation faible à nulle) à l'embouchure du delta de marée au sud de Jullouville entre 0 et $5 \mathrm{~m}$ de profondeur Fig. 2). Les enregistrements de la vitesse et de la direction des courants ont été acquis toutes les $30 \mathrm{~min}$, intégrés sur une période de $5 \mathrm{~min}$, à $1 \mathrm{~m}$ au-dessus du 


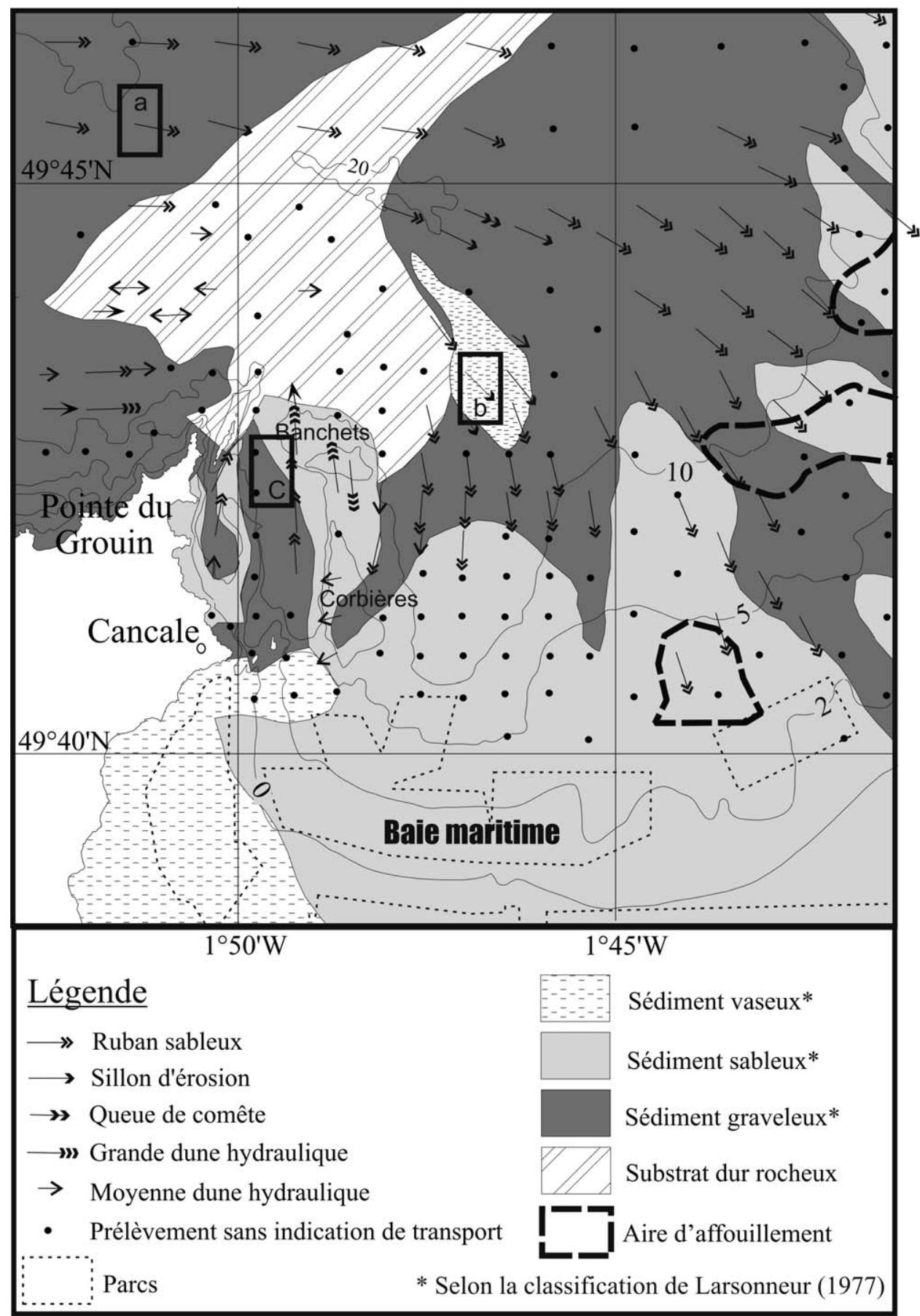

Fig. 3. Carte des transports sédimentaires dans la baie occidentale maritime à partir des levés au sonar à balayage latéral. Net sand transport in the occidental maritime zone of the Mont-Saint-Michel bay from side scan sonar interpretation.

fond, à mi-profondeur et à $1 \mathrm{~m}$ sous la surface. Les vitesses sont mesurées entre 0 et $350 \mathrm{~cm} \mathrm{~s}^{-1}$ avec une résolution de $0,2 \mathrm{~cm} \mathrm{~s}^{-1}$ et $0,5^{\circ}$ pour la direction.

\subsection{Principaux résultats de dynamique sédimentaire}

\subsubsection{La baie occidentale maritime}

Cette frange côtière de la baie est caractérisée par des séquences de dépôts sédimentaires superficiels tronquées mettant en contact et sans transition granulométrique les bancs sableux avec la roche et les sédiments grossiers parfois envasés dans les dépressions littorales Fig. 3. Au nord de la pointe du Grouin, les figures sédimentaires longitudinales constituées d'un faisceau dense de rubans de sables moyens à grossiers progressent vers l'est sur un reg hétéroclite, puis sont progressivement déviées, une fois le cap franchi, en direction du sud et du sud-ouest dans un grand mouvement tourbillonnaire horaire centré sur le banc des Corbières. Cette virgation des rubans de sable Fig. 4a et des sillons d'érosion pluridécamétriques Fig. 4b décrit la courbure d'un large vortex de $7 \mathrm{~km}$ de diamètre dont la limite orientale 

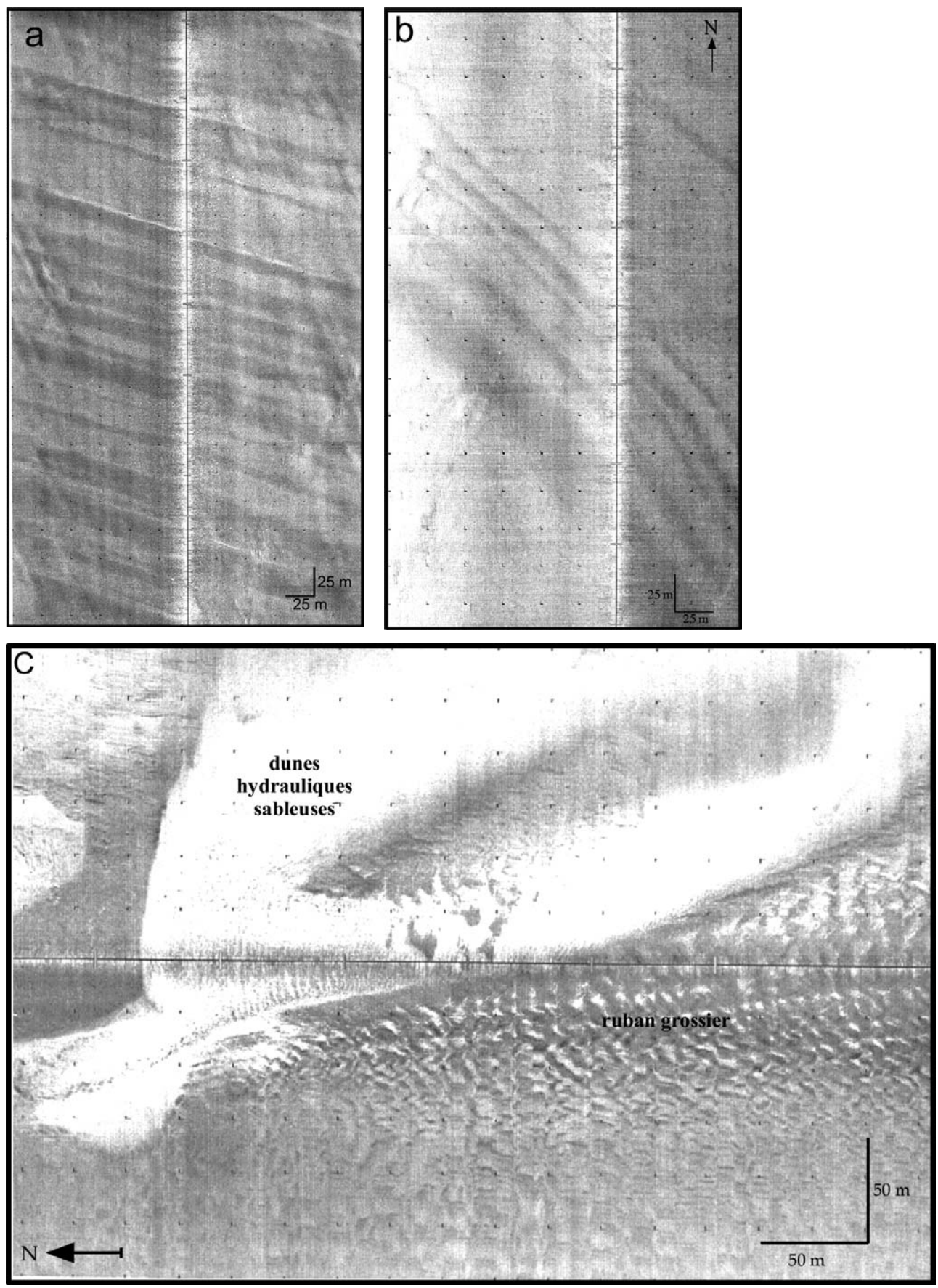

Fig. 4. A : exemple de rubans sableux ; B : exemple de sillons d'érosion ; C : exemple d'un ruban grossier. A: sonograph of sand ribbon; B: sonograph of longitudinal furrows; C: sonograph of coarse ribbon.

se situerait au droit de Cherrueix. La crête du banc des Corbières constitue une frontière hydrodynamique majeure séparant à l'est, l'action résiduelle et dominante des courants de flot, responsables de l'asymétrie du banc vers l'ouest et à la côte, ceux de jusant, chenalisés par les dépressions morphologiques et le banc du Chatry (Ehrhold, 1999). Cette divergence observée localement dans les directions sédimentaires, est également enregistrée à plus petite échelle dans les asymétries opposées nord et sud des grandes dunes hydrauliques des Banchets constituant la terminaison septentrionale du banc des Corbières. Entre Cancale et la pointe du Grouin, le long de la côte, les sédiments sont charriés vers le nord sous l'effet des puissants courants de vidange de cette partie de la baie (6 nœuds pour une marée de grande vive-eau). Ces vitesses engendrent sur le fond des figures sédimentaires particulières, à l'origine de la genèse des rubans sableux observée par McLean (1981) en canal expérimental et Braud (1986) à l'embouchure de la Gironde. Elles sont constituées de petites dunes hydrauliques lingoïdes ou en barkanes de longueur d'onde moyenne de 6 m environ, recoupées transversalement par des saignées rectilignes ou «longitudinal furrows » mettant à nu les graviers sous-jacents Fig. 4c. Le 
banc du Chatry accroché en bannière derrière la pointe de la Chaîne et la couverture de sable fin qui lui est associée, s'effilent vers le nord, modelés de petites dunes lingoïdes et découpés par un faisceau de sillons graveleux indiquant un transit sud-nord des sédiments. À quelques encablures de la côte et du fond de baie vaseux, la morphologie de ces figures sédimentaires témoigne d'un niveau hydrodynamique élevé sur le fond et d'un déficit en matériel sableux.

\subsubsection{La baie orientale estuarienne}

Les fonds plats du centre de la baie concentrent des faisceaux de rubans sableux coquilliers qui migrent vers l'est entre la pointe du Grouin et Chausey puis, au droit de la Chapelle Saint-Anne, bifurquent rapidement vers le sud-est en direction du delta de marée. Localement, correspondant aux zones dites des Petits Bancs ou de la Basse Parisienne Fig. 1, de grandes dunes hydrauliques isolées sont associées aux cortèges de figures longitudinales dont les vitesses de déplacement vers le fond de baie ont été estimées à une dizaine de mètres par an, c'est-à-dire de l'ordre de grandeur de celles mesurées par Berné (1991) au Nord de Chausey sur les dunes de Surtainville. À l'entrée de la baie orientale Fig. 5, les fortes et rapides variations de contraste de rétrodiffusion des sédiments montrent un prisme sableux bioclastique entaillé par trois larges couloirs morphosédimentaires grossiers composés de graviers bioclastiques et dans lesquels progressent les rubans sableux du large. Ils se connectent ainsi à la couverture sableuse continue et participent à l'engraissement du delta de marée. Le couloir nord longe le flanc nord du banc de Rondehaie jusqu'à la pointe rocheuse de Champeaux. Le couloir sud correspond à la position de l'ancien lit du Couesnon à l'extrémité duquel se sont établis les massifs d'Hermelles (L'Homer, 1988). À la côte, les sables roux carbonatés et bien triés sont entraînés latéralement, le long de la côte ouest du Cotentin entre Jullouville et Genêts. Cette dynamique sableuse est accentuée par la dérive littorale engendrée par les houles de tempête qui mettent à l'affleurement sur la plage des niveaux de tangues anciennes et plus au sud construisent de nombreuses flèches sableuses (Compain et al., 1988). Les prélèvements effectués sur le sommet du banc de Rondehaie quelques jours après une longue période de tempêtes de secteur ouest, présentent les caractéristiques d'un sédiment réduit bien trié et lavé dont on retrouve à plusieurs reprises la signature dans les prélèvements à l'embouchure du delta de marée.

Entre les biohermes à Hermelles et Jullouville, les vitesses maximales de courant enregistrées au moment du flot dans des conditions de marée de grande vive-eau, sont à $1 \mathrm{~m}$ au-dessus du fond de l'ordre de 80 à $100 \mathrm{~cm} \mathrm{~s}^{-1}$ Fig. 6. Elles dépassent très largement le seuil critique de mise en mouvement des particules sédimentaires de cette région (Migniot, 1992) et entraînent une remobilisation de la tranche superficielle de la couverture sédimentaire. Ce phénomène a été observé dans les carottages, sur les 15 premiers centimètres d'épaisseur, par l'érosion et le recouvrement de dépôts de basse énergie (sédiments vaseux) par des sables coquilliers ocres et bien triés (Ehrhold, 1999). Le delta de marée constitue un vaste réceptacle sédimentaire où les apports sableux du flot sont remobilisés et brassés par les courants de jusant.

\section{Modélisation des processus de charriage}

\subsection{Modèle hydrodynamique}

Le code de calcul utilisé est basé sur la résolution des équations de Saint-Venant par une méthode de différences finies. Plus précisément, on utilise une technique de pas fractionnaire (Nguyen et Ouahsine, 1997) pour traiter séparément les phénomènes de convection, diffusion et de propagation des ondes. L'étape de convection est traitée par une méthode des caractéristiques du quatrième ordre développée par Nguyen et Martin (1988). Cette technique minimise la diffusion numérique. En sortie du code, on récupère en chaque nœud du maillage le triplet $(H, U, V)$ représentant respectivement la hauteur d'eau et les composantes horizontales de la vitesse intégrée sur la colonne d'eau. Le domaine de calcul est limité à l'est par la côte Granville-Jullouville, au sud par la baie du Mont-Saint-Michel, à l'ouest par la longitude $1^{\circ} 55^{\prime} \mathrm{W}$ et enfin au nord par la latitude $48^{\circ} 55^{\prime} \mathrm{N}$. Un tiers de cette zone peut être exondé à marée basse, ce qui nécessite un traitement numérique spécifique (Guillou et al., 1997). Le domaine a été couvert par un maillage régulier de $150 \times 150$ cellules $(212 \times 188 \mathrm{~m})$. Aux frontières solides (côtes), une condition de non glissement est imposée. Aux frontières ouvertes, l'élévation de la surface libre est imposée par les résultats d'un calcul précédent fait à l'échelle du golfe normand-breton. La vitesse, quant à elle, est calculée à l'aide d'une condition de radiation. Le pas d'intégration des phénomènes hydrodynamiques est de $6 \mathrm{~s}$. Une sauvegarde de l'hydrodynamique est réalisée toutes les 12,4 min en temps de cycles. C'est cette hydrodynamique qui sert au calcul des débits solides instantanés (en direction et en intensité). Cette période correspond au pas d'intégration du calcul du charriage. Afin de se rapprocher des conditions hydrodynamiques naturelles à l'origine du déplacement potentiel des particules sédimentaires, nous avons pris en compte des conditions de marée de vive-eau pour un coefficient de marée de 70 et de grande vive-eau sans forçage atmosphérique (coefficient de marée de 103). Les conditions d'agitation par le vent et les houles n'ont donc pas été prises en compte dans les simulations.

\subsection{Paramètre de la formule de charriage}

La formule (Équation 1) de Bagnold modifiée par Gadd et al. (1978) est celle choisie pour calculer le transport solide par charriage. Cette formule lie le débit horizontal de matériel solide $Q_{\mathrm{s}}$ à la différence entre la vitesse du courant $U_{100}$ $\left(\mathrm{cm} \mathrm{s}^{-1}\right)$ et la vitesse critique $U_{100(\mathrm{CR})}\left(\mathrm{cm} \cdot \mathrm{s}^{-1}\right)$ à un mètre au-dessus du fond pour une granulométrie donnée.

$$
Q_{\mathrm{s}}=\beta\left(U_{100}-U_{100(\mathrm{CR})}\right)^{3}
$$




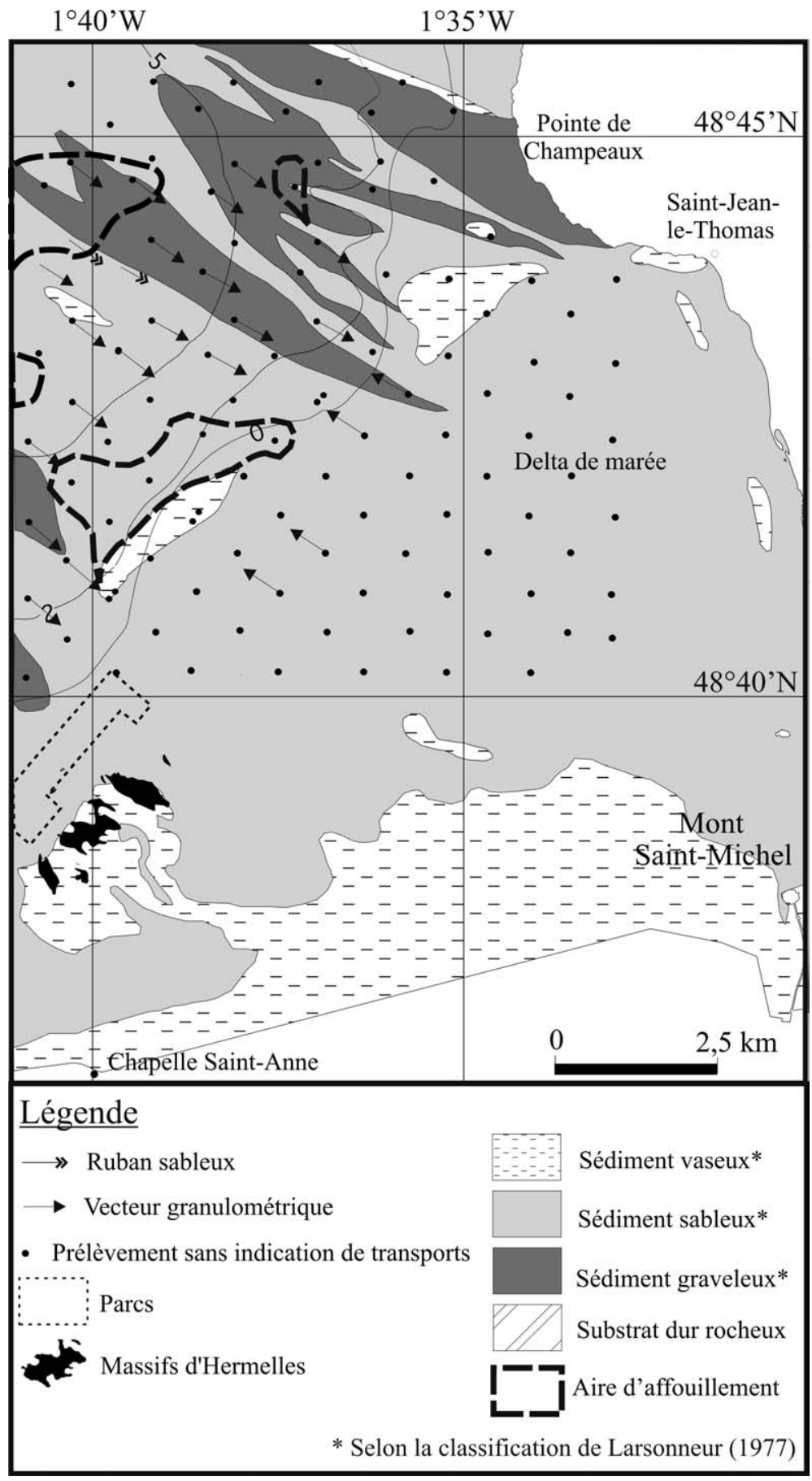

Fig. 5. Carte des transports sédimentaires dans la baie orientale estuarienne à partir des levés au sonar à balayage latéral et de l'interprétation des caractéristiques granulométriques des sédiments.

Net sand transport in the oriental estuarine zone of Mont-Saint-Michel bay from side scan sonar interpretation and sediment granulometry characteristic.

Dans cette équation, pour calculer $Q_{\mathrm{s}}$ seule la vitesse $U_{100}$ reste à définir, les autres paramètres sont des valeurs calculées pour différentes granulométries par Gadd et al. (1978), Langhorne (1981) et Dewez (1988). La vitesse critique de l'écoulement $U_{100(\mathrm{CR})}$ est le paramètre de seuil en deçà duquel il n'y a pas déplacement du sédiment. Le débit massique $\mathrm{Q}_{\mathrm{s}}$ s'annule lorsque $U_{100}<U_{100(\mathrm{CR}) .} \beta$ est un coefficient de proportionnalité dont la valeur est de $7,2210^{-5} \mathrm{~g} \mathrm{~cm}^{-4} \mathrm{~s}^{2}$ pour un grain moyen de $0,19 \mathrm{~mm}, 1,0210^{-5} \mathrm{~g} \mathrm{~cm}^{-4} \mathrm{~s}^{2}$ pour un grain moyen de $0,32 \mathrm{~mm}$ et $1,7310^{-5} \mathrm{~g} \mathrm{~cm}^{-4} \mathrm{~s}^{2}$ pour un grain moyen de $0,45 \mathrm{~mm}$. Les paramètres $U_{100(\mathrm{CR})}$ et $\beta$ (Gadd et al., 1978 ; Hearthershaw, 1981 ; Langhorne, 1981 ; Dewez, 1988) dépendent de la nature des sédiments et de la morphologie du fond, tandis que $\mathrm{U}_{100}$ dépend de l'hydrodynamique. Dans cette étude, $\beta$ et $U_{100(\mathrm{CR})}$ n'ont pas été calculés à partir du diamètre moyen des sédiments $D_{50}(\mathrm{~mm})$ mais en fonction du mode principal de la fraction sableuse des sédiments prélevés dans la baie (Ehrhold, 1999 ; Caline et al., 1982) et à 

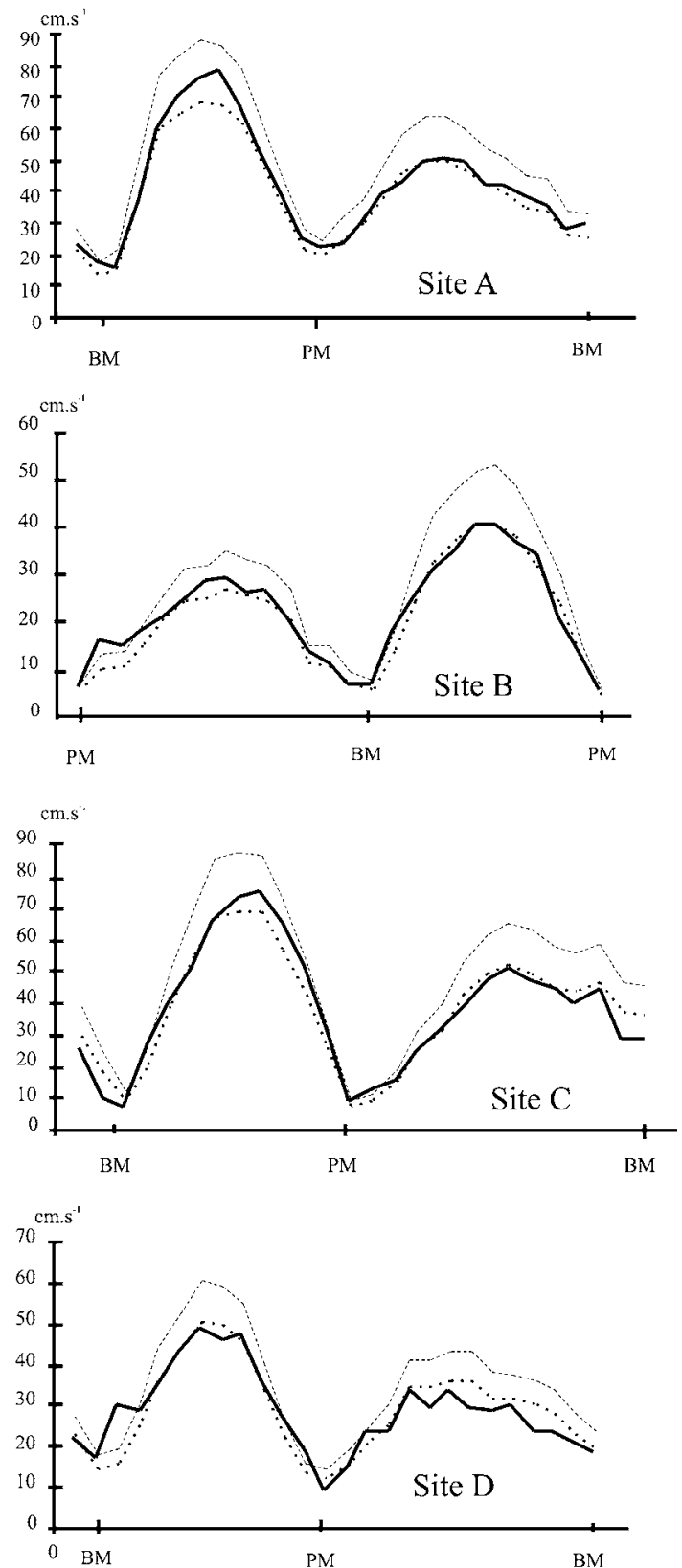

Mesurée à $1 \mathrm{~m}$ au-dessus du fond

$$
\mathrm{Z}_{0}=0,6
$$$$
\mathrm{z}_{\mathrm{o}}=0,1
$$

Fig. 6. Influence de la rugosité $z_{0}$ sur le calcul de la vitesse théorique $U_{100}$. Sensitivity of the current estimation $1 \mathrm{~m}$ above the bed for different values of seabed roughness $z_{0}$.

partir des travaux d'Hearthershaw (1981). En effet $40 \%$ des échantillons prélevés dans le domaine d'étude sont des sédiments grossiers dont la médiane est supérieure à $2 \mathrm{~mm}$ (Ehrhold, 1999). Or les profils au sonar à balayage latéral ont montré l'importance de la dynamique sableuse en charriage sous forme de rubans et de voiles superficiels sur ces fonds grossiers entre la côte bretonne et l'archipel des îles Chausey (Ehrhold et al., 1997 ; Ehrhold, 1999). Le diamètre moyen des sédiments très grossiers contenant 70 à $80 \%$ de grains supérieurs à $2 \mathrm{~mm}$ occulte le rôle de la fraction sableuse dans la quantité de matériels sédimentaires susceptibles d'être transportés.

\subsection{Méthodologie de calcul des flux sédimentaires}

Il est couramment admis qu'un écoulement turbulent à proximité du fond dans un liquide non stratifié, suit sur la verticale une loi logarithmique simple et universelle (Dyer, 1986) : la loi de von Kármán-Prandtl (Équation 2).

$$
\frac{U_{\mathrm{Z}}}{\mathrm{U}_{*}}=\frac{1}{\mathrm{~K}} \cdot \ln \left(\frac{\mathrm{z}}{\mathrm{z}_{0}}\right)
$$

$\mathrm{U}_{\mathrm{z}}$ est la vitesse des courants à une hauteur $\mathrm{z}$ au-dessus du fond, $\mathrm{U}^{*}$ la vitesse de frottement, $z_{0}$ la rugosité du fond et $\mathrm{K}$ la constante de von Kármán-Prandtl égale à 0,4 (Dyer, 1986). La méthodologie appliquée pour relier la vitesse $\hat{U}$ intégrée sur la colonne d'eau $H$ et calculée par le modèle, à la vitesse $U_{\mathrm{z}}$ à une hauteur $z$ au-dessus du fond, s'appuie sur la méthodologie de Grochoswski et al. (1993) dans la prédiction des flux sédimentaires à l'échelle de la Manche. $\hat{U}$ se substitue à $U_{\mathrm{z}}$ en considérant l'approximation que la vitesse moyenne calculée par le modèle $\hat{U}$ suit le profil logarithmique mais sur toute la tranche d'eau définie par $H$ (Équation 3). Grochoswski et al. (1993) montrent que lorsque $U_{\mathrm{z}}=\hat{U}$ alors $z / H=\mathrm{e}^{-1} \approx 0,368$.

$$
\frac{\hat{U}}{\mathrm{U}_{*}}=\frac{1}{\mathrm{~K}} \cdot \ln \left(\frac{H}{\mathrm{ez}_{0}}\right) \quad \text { avec } \quad 1 / \mathrm{e}=0,368
$$

Ainsi $U_{100}$ variera pour une valeur donnée de $\hat{U}$ et $H$ seulement en fonction de $z_{0}$. Cette approximation a été calculée par Grochoswski et al. (1993) sur la base de mesures faîtes à différents niveaux par des courantomètres et par Ehrhold (1999) à $1 \mathrm{~m}$ au-dessus du fond Fig. 6. Elle est elle-même fonction des approximations faîtes sur $z_{0}$ mais peut être admise avec $95 \%$ de niveau de confiance pour $72 \%$ du temps et $85 \%$ de niveau de confiance pour $86 \%$ du temps. En réalité les écarts deviennent importants au moment des pics de flot et de jusant, c'est-à-dire pendant les périodes où le sédiment est le plus mobilisé Fig. 6. De l'Équation 3, la vitesse de frottement $U_{*}$ est calculée et reliée à $U_{100}$ par la relation de Dyer (1986) (Équation 4).

$$
\tau=\mathrm{C}_{100} \rho U_{100}^{2} \text { avec } \quad \mathrm{U}_{*}=(\tau / \rho)^{1 / 2}
$$

$\mathrm{C}_{100}$ est un coefficient de traînée dont la valeur a été fixée à 0,003 correspondant à des fonds où l'amplitude des rides est inférieure à $20 \mathrm{~cm}$ et les vitesses à $1 \mathrm{~m}$ au-dessus du fond supérieures à $15 \mathrm{~cm} \mathrm{~s}^{-1}$ (Sternberg, 1972).

Une synthèse des valeurs de $z_{0}$ disponibles à partir de différentes études (Grochoswski et al., 1993 ; Ehrhold, 1999) est présentée en Tableau 1 Celles-ci varient de 0,05 pour les vases, de 0,3 à 0,6 pour les sables et de 0,3 à 0,45 pour les sédiments grossiers (graviers et cailloutis). Les valeurs de $z_{0}$ ont été définies pour tous les prélèvements à partir de ce tableau sur la base des analyses granulométriques (Ehrhold, 1999) et estimées en domaine intertidal à partir des cartes sédimentaires existantes (Caline et al., 1982 ; Larsonneur, 1989). 
Tableau 1

Exemples de valeurs significatives de rugosité $z_{0}$ selon la classification de Larsonneur (1977). Synthèse d'après Grochowski et al. (1993), Heathershaw (1981), Lees (1983), Soulsby (1983) $z_{0}$ roughness length values corresponding to Larsonneur (1977) sedimentary classification. (compilation of Grochowski et al. (1993), Heathershaw (1981), Lees (1983) and Soulsby (1983) studies)

\begin{tabular}{|c|c|c|c|}
\hline Classification & Nature du sédiment & $z_{0}(\mathrm{~cm})$ & Morphologie du fond \\
\hline \multicolumn{4}{|c|}{ Larsonneur (1977) } \\
\hline $\mathrm{V}$ & Vase ou Sable vaseux & 0,05 & Fond plat \\
\hline $\mathrm{Se}$ & Sable très fin & 0,1 & \multirow{4}{*}{$\begin{array}{l}\text { Fond plat, ridé ou avec de grandes } \\
\text { structures sableuses }\end{array}$} \\
\hline $\mathrm{Sd}$ & Sable fin & 0,3 à 0,5 & \\
\hline $\mathrm{Sc}$ & Sable moyen & 0,6 & \\
\hline $\mathrm{Sb}$ & Sable grossier & 0,6 & \\
\hline $\mathrm{Gb}$ & Gravier & 0,3 & \multirow{4}{*}{$\begin{array}{l}\text { Fond plat ou figures sédimentaires de } \\
\text { petite à grande longueur d'onde }\end{array}$} \\
\hline $\mathrm{Ga}$ & Gravier et cailloutis & 0,3 & \\
\hline $\mathrm{Cb}$ & Cailloutis et gravier & 0,32 & \\
\hline $\mathrm{Ca}$ & Cailloutis et galet & 0,45 & \\
\hline
\end{tabular}

Le code de calcul donne les 2 composantes de la vitesse ainsi que la hauteur de la colonne d'eau toutes les 12,4 min. Nous calculons donc les débits solides toutes les 12,4 min. Il est alors possible de calculer l'intensité de la vitesse $\hat{U}$ (Équation 2), ainsi que l'angle instantané $\theta$ de direction de l'écoulement. L'Équation 1 permet de déterminer l'intensité du débit sédimentaire $Q_{\mathrm{s}}$ en tous points du maillage en connaissant les valeurs de $\hat{U}$ et $h$, ainsi que $C_{100}, \mathrm{z}_{\mathrm{o}}$ et $\beta$ interpolés en chacun de ces points par triangulation. La direction instantanée de déplacement des sédiments est celle des masses d'eau. Elle est représentée par $\theta$. Elle est donc considérée constante sur toute la colonne d'eau. De là, le débit vectoriel sédimentaire $Q_{\mathrm{v}}$ peut être déterminé. C'est ce dernier débit qui par une intégration sur un cycle de marée est représenté sur les Figs. 7 et 8.

\subsection{Résultats}

Les résultats des simulations des flux résiduels et potentiels $Q_{\mathrm{v}}$ de sédiments charriés (exprimés en tonne par mètre durant un cycle de marée) sont présentés Figs. 7 et 8 pour des coefficients de marée de 70 et 103. En raison de la grande variabilité de ces flux sur l'ensemble du secteur étudié, il est apparu nécessaire de les différencier suivant 4 classes de même dimension séparées d'un facteur 10. Ceci facilite non seulement la lecture des documents, mais aussi l'intérêt des informations fournies par les flux les plus faibles.

\subsubsection{La baie occidentale maritime}

Dans les 2 simulations, les sédiments charriés pénètrent dans la baie depuis les fonds grossiers au Nord de la pointe du

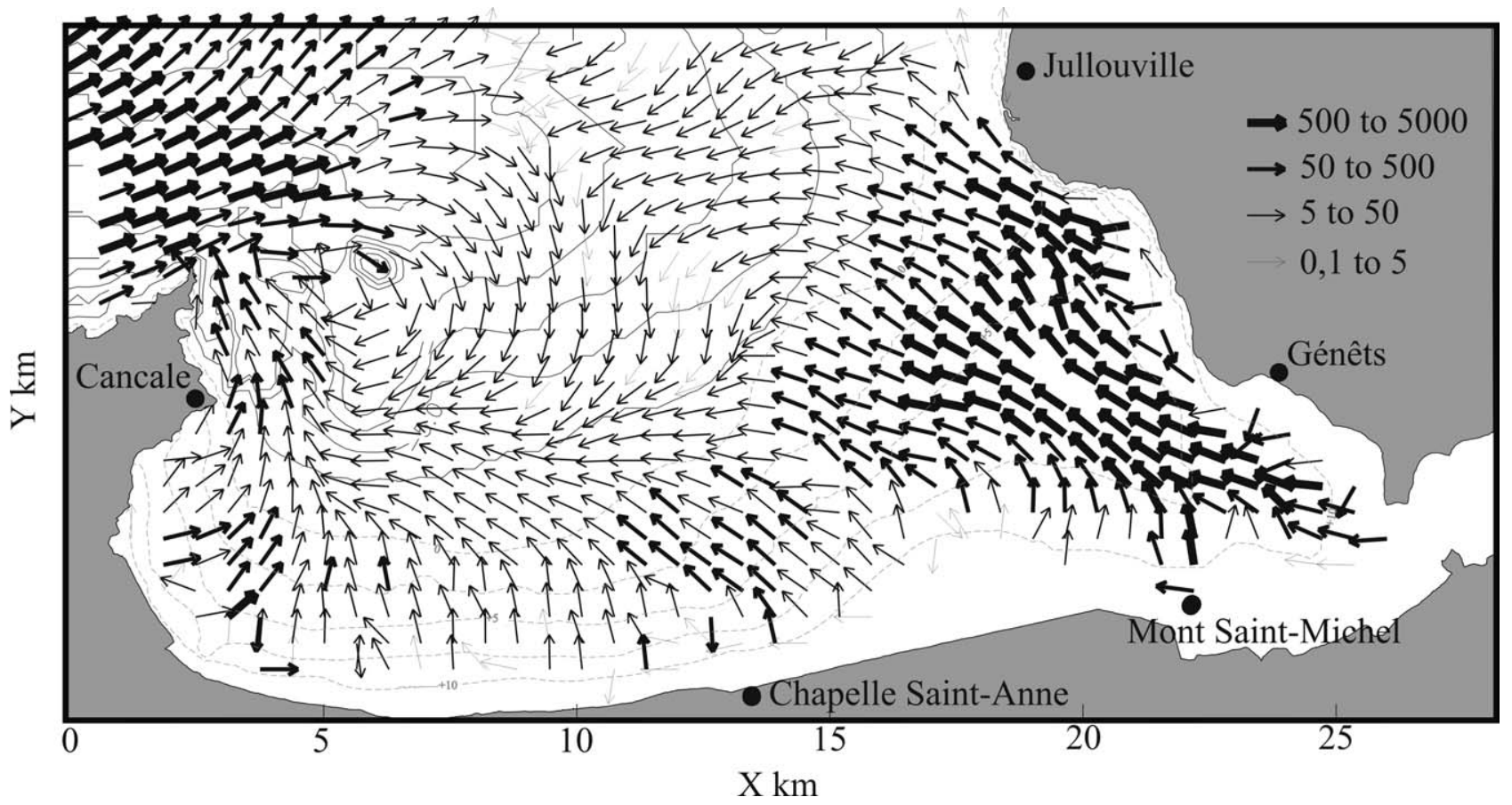

Fig. 7. Flux de sédiments charriés $\left(\mathrm{t} \mathrm{m}^{-1}\right.$ marée $\left.^{-1}\right)$ en baie du Mont-Saint-Michel pour un coefficient de marée de 70. Bedload sediment discharge ( $\mathrm{m}^{-1}$ marée- $\left.^{1}\right)$ in the Mont-Saint-Michel bay for a tidal coefficient of 70 . 


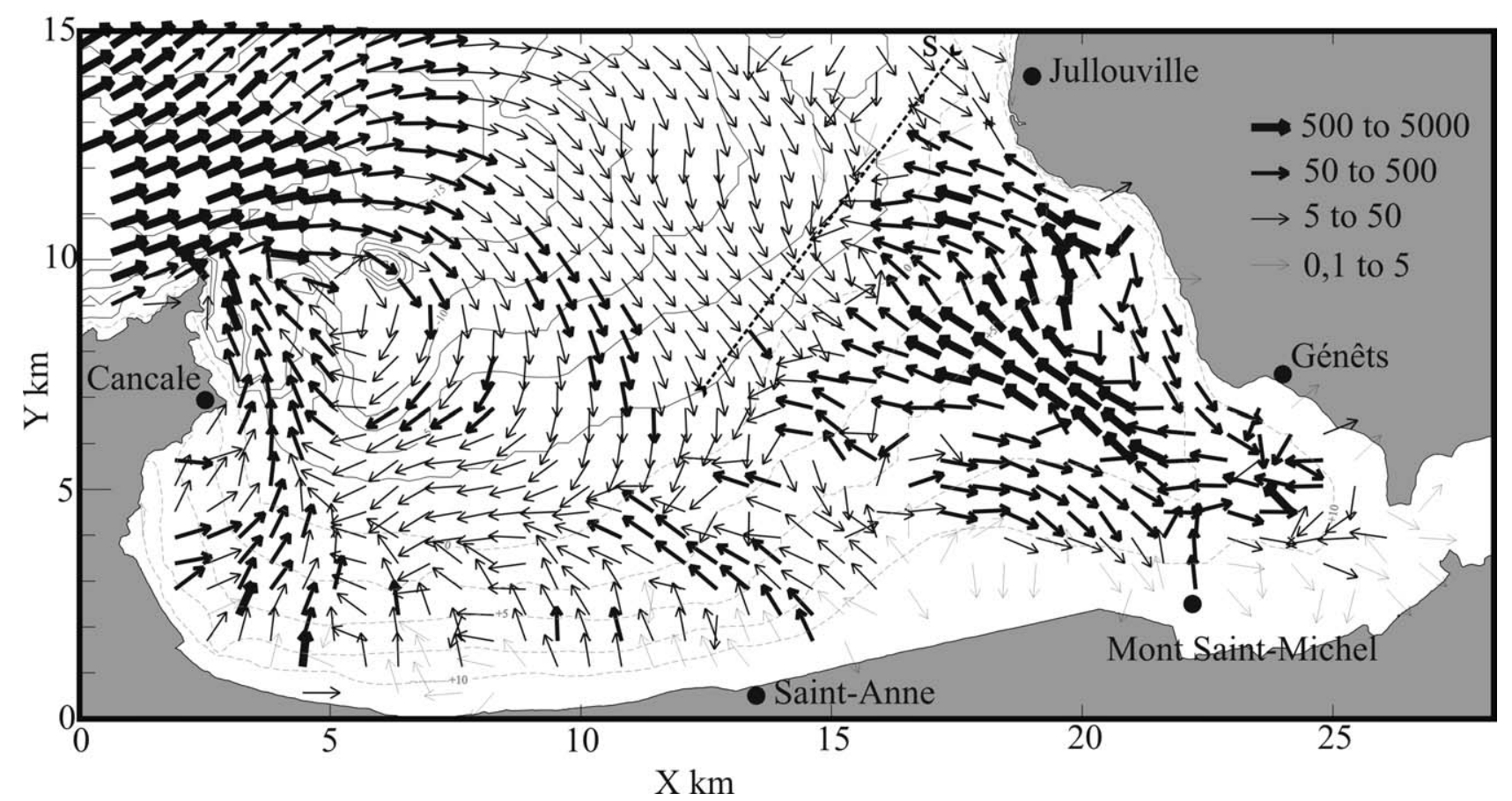

Fig. 8. Flux de sédiments charriés (t. $\mathrm{m}^{-1}$ marée $\left.{ }^{-1}\right)$ en baie du Mont-Saint-Michel pour un coefficient de marée de 103. Bedload sediment discharge ( $\mathrm{t} \mathrm{m}^{-1}$ marée $\left.{ }^{-1}\right)$ in the Mont-Saint-Michel bay for a tidal coefficient of 103.

Grouin en décrivant un large vortex horaire amplifié pour une marée de grande vive-eau. Dans ces conditions, il occupe tout l'espace de la baie maritime et coïncide exactement avec la rotation des transports sédimentaires observés sur le fond Fig. 3. Sa limite occidentale au large de Cherrueix, correspond à un changement majeur dans la répartition et la nature des dépôts sédimentaires, aussi bien en domaine intertidal (Caline et al., 1982) que subtidal (Ehrhold, 1999).

Sur l'estran, quel que soit le coefficient de marée, aucune remontée du sédiment depuis le large n'est constatée. Si en effet les taux de sédimentation relevés par Berger et Caline (1991) et Walker (2001) y sont très faibles, de l'ordre de quelques centimètres par an $(0,4$ à $1 \mathrm{~cm} / \mathrm{an})$ et sans aucune mesure avec les variations décimétriques à métriques des grèves de la baie orientale (Larsonneur, 1989), ces auteurs ne montrent pas, pour autant, un démaigrissement des plages vaseuses et sablovaseuses de cette partie de la baie. On peut expliquer ce désaccord par la non-prise en compte des effets de houle dans le calcul. En effet, la dynamique sédimentaire intertidale y est contrôlée par l'action des houles de tempêtes qui poussent les cordons coquilliers vers le haut-estran (Bonnot-Courtois, 1994 ; Larsonneur, 1989).

\subsubsection{La baie orientale estuarienne}

Inversement, les 2 simulations donnent dans cette partie de la baie, une image différente de la circulation résiduelle des sédiments. En vive-eau moyenne Fig. 7, les flux subtidaux prolongent les flux sortant de jusant en zone intertidale, puis migrent vers l'ouest pour se connecter au vortex de la baie occidentale. Or les échanges Est-Ouest suggérés par ce scénario n'ont jamais été validés dans cette région que ce soit en surface par les lâchés de flotteurs (Larsonneur, 1989) ou sur le fond par la direction nord-ouest sud-est des figures sédimentaires longitudinales observées au sonar à balayage latéral.

L'image des transports obtenue pour la simulation d'un fort coefficient Fig. 8 semble plus conforme à la connaissance des transports sédimentaires établie sur la base des données d'acoustique sous-marine au large et des vecteurs de transport granulométrique en domaine intertidal Fig. 5. Ces derniers ont été définis sur l'ensemble de la baie (Ehrhold, 1999) en combinant 2 facteurs : l'évolution de la couleur en tant que marqueur de la dynamique sédimentaire et l'indice de classement qui s'améliore dans la direction de transport. Les flux de secteur nord-ouest convergent vers l'embouchure du delta de marée au niveau du zéro hydrographique et sont localement réfractés au large de Jullouville en remontant le flanc long du banc de Rondehaie vers sa crête. Ce phénomène de déviation progressive des transports sédimentaires depuis le pied du banc jusqu'à son sommet est un des éléments moteurs de la dynamique maintenant bien connue de ce type de figures sédimentaires (Dewez, 1988). Les flux en charriage pénètrent dans la partie interne de la baie (domaine pré-estuarien), soit par le nord en longeant la côte entre Champeaux et Genêts, soit à l'est du massif des Hermelles. Ces 2 voies d'apports sédimentaires coïncident avec la terminaison des 2 couloirs grossiers décrits par l'étude sédimentologique et acoustique.

Le couloir sud s'oriente progressivement vers l'est en direction du Mont-Saint-Michel sur une largeur de plus de $2 \mathrm{~km}$. Dans ce couloir, l'estran est modelé par de grandes mégarides rectilignes souvent anastomosées dont l'asymétrie à marée basse est orientée à l'Est. 
Tableau 2

Exemples de débits massiques sédimentaires calculés sur la base de l'équation de Bagnold modifiée par Gadd et al. (1978). (SF, sable fin ; GVE, grande vive-eau ; VE, vive-eau ; ME, morte-eau).Examples of sand transport rates defined by Bagnold's formula modified by Gadd et al. (1978) (SF, Fine sand; GVE, large spring tide; VE, spring tide; ME, low tide)

\begin{tabular}{|c|c|c|c|c|}
\hline Sites/Références & Grain moyen $(\mathrm{mm})$ & Vitesse Max $(\mathrm{cm} / \mathrm{s})$ & Coefficient de marée & $Q_{\mathrm{v}}\left(\mathrm{t} \mathrm{m}^{-1}\right.$ maré $\left.^{-1}\right)$ \\
\hline Site A & 1,777 & 78 & 107 & 0,903 \\
\hline Site B & 0,945 & 40 & 52 & 0,197 \\
\hline Site C & 1,820 & 74 & 100 & 4,992 \\
\hline Site D & 0,365 & 49 & 85 & 0,094 \\
\hline
\end{tabular}

Le couloir nord entre Champeaux et Genêts est contraint par la migration latérale et saisonnière d'un chenal de la Sée-Sélune pouvant être à l'origine d'une érosion très active de la plage. Il participe avec la dérive littorale (Compain et al., 1988) à la constitution de cordons de déferlements arqués progradants vers le sud et à l'engraissement progressif de ce secteur. Ces faciès sédimentaires de haut-estran et de haute énergie caractérisent de forts apports de sable et une participation déterminante de la dynamique tidale.

Les simulations montrent que le corps principal du delta de marée est dominé par des flux résiduels sortants. Le modèle met ainsi en exergue le rôle des nombreux chenaux de marée qui canalisent les courants de vidange de la baie dans l'effet de chasse des sédiments au moment du retrait des eaux marines. Le rôle de ces chenaux est fondamental dans le contrôle des vitesses de sédimentation dans ce secteur (Larsonneur, 1989). Les essais de mesures des taux de sédimentation sur le terrain et des débits solides se sont soldés par un recouvrement ou une dispersion des traceurs dans cet environnement où le brassage sédimentaire est important à l'échelle d'une marée (Doulcier, 1977). La zone de confrontation avec les flux venant du large est caractérisée par des cellules antihoraires à l'emplacement de vasières actuelles Fig. 3, également décrites par Doulcier (1977) mais à proximité du Mont-Saint-Michel. Il s'agit d'un secteur où localement la colonne d'eau peut montrer une stratification et où la direction du transport sédimentaire n'est pas toujours colinéaire à la direction du courant d'intensité maximum. Le modèle 2DH de calcul utilisé dans cette étude ne prend pas en compte ces phénomènes tridimensionnels.

\subsubsection{Les débits massiques}

L'échelle des flux théoriques et potentiels varie de quelques dixièmes de tonnes à plusieurs milliers de tonnes de sables par mètre linéaire et par marée. Sur les stations de mesures en point fixe Fig. 2, ils ont été comparés aux débits résiduels calculés selon la même méthode mais à partir des données des courantomètres Fig. 6et Tableau 2). Les débits calculés à partir des mesures varient d'une centaine de kilos à 5 tonnes pour des vitesses de $75 \mathrm{~cm} \mathrm{~s}^{-1}$ et des coefficients supérieurs à 100. Ils sont tous orientés dans la direction des courants de flot et pour des vitesses de courants équivalentes, de l'ordre de grandeur des débits estimés par Langhorne (1981) sur une dune hydraulique sableuse $\left(3,3 \mathrm{t} \mathrm{m}^{-1}\right.$ marée $\left.^{-1}\right)$ ou à ceux calculés sur les fonds sableux de la baie de Swansea à partir des expériences de traceurs radioactifs $\left(1,3 \mathrm{t} \mathrm{m}^{-1}\right.$ marée $^{-1}$; Heathershaw, 1981). Larsonneur et Walker (1986) estiment à partir de leur modèle sur le golfe normand-breton, des valeurs minima de $4,5 \mathrm{t} \mathrm{m}^{-1}$ marée ${ }^{-1}$ dans la région du delta de marée ainsi qu'au Nord de la pointe du Grouin et de 1,4 à 2,6 t m marée $^{-1}$ pour le reste de la baie. À une autre échelle et en prenant en compte le diamètre moyen $D_{50}$ (mm), Grochowski et al. (1993) estiment à $0,45 \mathrm{t} \mathrm{m}^{-1}$ marée $^{-1}$ les flux les plus élevés dans toute la baie. À l'exception du site $\mathrm{C}$ et $\mathrm{D}$, il semble donc que les flux résiduels calculés par le modèle soient largement surestimés. Les plus fortes valeurs entre 50 et $5000 \mathrm{t}$ s'observent dans les secteurs où les courants sont canalisés dans le delta de marée, à l'Est de Cancale et au Nord de la pointe du Grouin. Or, la signification de ces débits n'est pas la même selon la nature de la couverture sédimentaire superficielle. En effet, la quantité de sable potentiellement en transit dépend de la présence de ce matériel dans le sédiment. Elle varie du simple au triple. L'importance de la fraction sableuse est bien moindre, 32 contre $80 \%$ en moyenne du poids du sédiment, respectivement dans les sédiments grossiers de la passe entre les îles Chausey et la pointe du Grouin Fig. 3 que dans ceux du prisme côtier sableux Fig. 5. Cet effet se traduit à l'ouest par une dynamique sédimentaire sableuse très superficielle, localisée sous la forme de rubans de sable (Figs. 3a et 3b) et dont les intensités sont très probablement surestimées par le modèle.

Les valeurs des débits massiques des sites $\mathrm{A}$ et $\mathrm{C}$ Tableau 2 pour un grain moyen correspondant à un sable grossier $(1,8 \mathrm{~mm})$ et des conditions de marnage sensiblement égales montrent que la vitesse maximale instantanée $U_{\max }$, respectivement 78 et $74 \mathrm{~cm} \mathrm{~s}^{-1}$, ne reflète pas toujours le débit solide maximum. L'inégalité de la durée des périodes de flot et de jusant durant lesquelles $U_{\mathrm{z}}$ est supérieure à la vitesse critique $U_{\mathrm{cr}}$ est à l'origine de la différence (facteur 5) des débits solides calculés ici à l'entrée du delta de marée. Ces variations locales peuvent s'expliquer par une morphologie du fond très différente avec pour le site $\mathrm{A}$, un fond relativement plat remontant en pente douce et pour le site $\mathrm{C}$, un effet canalisant par la présence du banc de Rondehaie et la proximité de la pointe de Champeaux. Sur la section transversale de $14 \mathrm{~km}$ de longueur entre Jullouville et le massif des Hermelles Fig. 8, le volume de sables charriés entrant annuellement dans le système du delta, a été extrapolé à $500000 \mathrm{~m}^{3}$ environ auquel s'ajoutent $900000 \mathrm{~m}^{3} \mathrm{an}^{-1} \mathrm{de}$ matières en suspension estimés à travers cette radiale à partir des filtrations d'eau de mer sur les mêmes stations (Ehrhold, 1999). Ces volumes participent au bilan positif de la sédimentation et au colmatage progressif de cette partie de la baie 
estimé à 1,3 millions de $\mathrm{m}^{3} \mathrm{an}^{-1}$ à partir des données de carottages et des coupes sismiques (Auffret in Larsonneur, 1989).

\section{Conclusion}

Dans cette note, une étude du transport de sédiment noncohésif par charriage en Baie du Mont-Saint-Michel a été réalisée en associant un modèle numérique 2DH et un échantillonnage fin de données sédimentaires.

Les directions des flux sédimentaires apparaissent en bon accord avec les relevés au sonar à balayage latéral, notamment pour les conditions de vive-eau d'équinoxe dans la baie orientale. Le modèle reproduit dès le coefficient d'une viveeau moyenne, la virgation des flux selon un grand tourbillon horaire centré sur la baie maritime et la convergence des transports sédimentaire vers la baie orientale depuis les fonds du large. Ces 2 cellules sont séparées par une frontière hydrosédimentaire au droit de la Chapelle Saint-Anne. Comme pour les flux de matières en suspension (Ehrhold, 1999), les résultats des simulations montrent que l'amplitude de la marée est un facteur prépondérant dans l'intensité des mouvements sédimentaires. Les périodes de colmatage du fond de la baie orientale par les sables charriés, seraient associées aux conditions de marnage extrêmes qui représentent $10 \%$ des marées sur une année. Les flux entrants dans le delta de marée progressent le long de couloirs géomorphologiques nord et sud parfaitement identifiés par leur signature acoustique. Cependant, le fait de ne pas intégrer les phénomènes instationnaires à périodes caractéristiques plus faibles telle que la houle classique ou de tempête, explique les divergences entre les observations et le calcul sur l'estran de la baie maritime. On a pu observé également au jusant, que les directions des courants de marée mesurées à $1 \mathrm{~m}$ sous la surface et à $1 \mathrm{~m}$ au-dessus du fond sur les sites $\mathrm{B}$ et $\mathrm{C}$, peuvent s'écarter jusqu'à des valeurs de $90^{\circ}$. Ces phénomènes renforcent dans cette zone l'intérêt d'inclure les effets tridimensionnels liés à une morphologie sous-marine et une configuration de la côte complexes. Une bathymétrie actualisée plus fine que celle réalisée par EDF en 1959 et qui demeure la seule disponible affinerait les résultats.

L'ordre de grandeur du colmatage du fond de baie orientale, estimé à partir des résultats des simulations, est de $500000 \mathrm{~m}^{3} \mathrm{an}^{-1}$ de sédiments charriés. Les débits charriés sont considérables sur la couverture sableuse continue et reflètent les forces hydrodynamiques en présence avec des vitesses de courants atteignant une centaine de centimètres par seconde à $1 \mathrm{~m}$ au-dessus du fond. Toutefois, les écarts avec les mesures de terrain peuvent être importants (facteur 10) et montrent les limites de l'utilisation des formules empiriques de calcul des débits solides malgré un effort de paramétrage à partir d'un semis important de données de terrain. Les paramètres empiriques comme la rugosité et le coefficient de traînée $\mathrm{C}_{100}$, peuvent introduire de grandes approximations dans le calcul des flux charriés. Une mauvaise évaluation de ces paramètres entraîne des erreurs sur l'estimation de la vitesse critique de mise en mouvement et fausse les débits résultants au moment des pics de flot ou de jusant. Cayocca (1996) montre qu'une erreur sur $z_{0}$ d'un facteur 10 entraîne une erreur de 40 \% sur $U_{*}$ soit, si le débit solide est une fonction cubique de la vitesse de frottement, une erreur de $175 \%$ sur celui-ci. On constate par ailleurs qu'une seule valeur de $z_{0}$ ne s'applique pas à toute la période de la marée pour un même site Fig. 6 .

L'association d'un modèle numérique à maille fine et d'un canevas sédimentaire dense telle qu'elle a été réalisée dans cette étude permet d'apporter de nouveaux éléments sur l'origine des flux sédimentaires dans la région de la baie du Mont-Saint-Michel. Il s'agit d'une première étape qui doit conduire à intégrer dans les futures simulations, des périodes tidales plus longues et des forçages atmosphériques.

\section{Remerciements}

Les auteurs tiennent à remercier le service hydrographique et océanographique de la marine à Brest, la région Basse-Normandie et les équipages de la flotte de l'Insu pour leur soutien.

Abréviations mathématiques :

$Q_{\mathrm{s}}$, débit massique instantané par section et par seconde ( $\mathrm{g}$ $\left.\mathrm{cm}^{-1} \mathrm{~s}^{-1}\right)$;

$U_{100}$, vitesse du courant à $1 \mathrm{~m}$ au-dessus du fond $\left(\mathrm{cm} \mathrm{s}^{-1}\right)$; $U_{100(\mathrm{CR})}$, vitesse critique de mise en mouvement du sédiment à $1 \mathrm{~m}$ au-dessus du fond $\left(\mathrm{cm} \mathrm{s}^{-1}\right) ; \beta$, coefficient de proportionnalité $\left(\mathrm{g} \mathrm{cm}^{-4} \mathrm{~s}^{2}\right) ; \hat{U}$, vitesse intégrée sur la hauteur d'eau $\mathrm{h}\left(\mathrm{cm} \mathrm{s}^{-1}\right) ; U_{z}$, vitesse du courant à une hauteur $z$ au-dessus du fond $\left(\mathrm{cm} \mathrm{s}^{-1}\right) ; U_{*}$, vitesse de frottement au-dessus du fond $\left(\mathrm{cm} \mathrm{s}^{-1}\right)$; K, Constante de von Kármán-Prandtl ; $z$, hauteur au-dessus du fond $(\mathrm{cm}) ; z_{0}$, rugosité du fond $(\mathrm{cm}) ; H$, hauteur de la colonne d'eau $(\mathrm{cm}) ;$ e, coefficient de régression ; $\mathrm{C}_{100}$, coefficient de traînée (drag coefficient) ; $\beta$, contrainte tangentielle $\left(\mathrm{g} \mathrm{cm} \mathrm{s}^{-2}\right)$; $\hat{\mathrm{U}}$, masse volumique du liquide $(\mathrm{g}$ $\left.\mathrm{cm}^{-3}\right) ; \theta$, angle de direction du courant $; U, V$, composantes de la vitesse; $Q_{\mathrm{v}}$, Débit massique vectoriel $\left(\mathrm{t} \mathrm{m}^{-1}\right.$ marée $\left.^{-1}\right)$.

\section{Références}

Allen, J.R.L., 1984. Principles of physical sedimentology. George Allen \& Unwin.

Belderson, R.H., Kenyon, N.H., Stride, A.H., Stubbs, A.R., 1972. Sonographs of the Sea floor. Elsevier,, Amsterdam.

Berné, S., 1991. Architecture et dynamique des dunes tidales : exemples de la marge atlantique française. université de Lille I Thèse de doctorat.

Berger, W., Caline, B., 1991. Estimation of sedimentation rates in the Bay of Mont-Saint-Michel (France) by ${ }^{210} \mathrm{~Pb}$ dating technique, a pilot study. Oceanol. Acta 11, 137-142.

Bonnot-Courtois, C., 1994. Les bancs coquilliers du haut estran de la baie du Mont-Saint-Michel entre Saint-Benoit des Ondes et la Chapelle SainteAnne Rapport d'étude EPHE/DREB.

Braud, F., 1986. La dynamique sédimentaire à l'embouchure d'un estuaire (intérêt du sonar latéral à l'étude de la passe Sud de la gironde). université de Bordeaux I Thèse de doctorat. 
Caline, B., Larsonneur, C., l'Homer, A., 1982. La Baie du Mont-SaintMichel : Principaux environnements sédimentaires. Livre jubilaire Gabriel Lucas, Mém. Géol. université de Dijon, pp. 37-51.

Compain, P., Larsonneur, C., Walker, P., 1988. Les sédiments et leur dynamique dans la partie Nord-Est de la baie du Mont-Saint-Michel. Bull. Soc. Linn. de Normandie 112-113, 109-114.

Cayocca, F., 1996. Modélisation morphodynamique d'une embouchure tidale : application aux passes d'entrée du bassin d'Arcachon. université de Bordeaux I Thèse de doctorat.

Dewez, S., 1988. Sédimentation et dynamique en Manche Orientale (de la baie d'Authie au Cap d'Aprech). université de Lille I Thèse de doctorat.

Doulcier, P., 1977. Le Mont-Saint-Michel. Le problème du caractère maritime du site. université de Paris XI Thèse Docteur-Ingénieur.

Dyer, K.R., 1986. Coastal and estuarine sediment dynamics. John Wiley \& sons. a Wiley interscience publication.

Ehrhold, A., Guillou, S., Auffret, J.P., Nguyen, K.D., 1997. Bedload transport predictions for the Mont-Saint-Michel's bay, using numerical model. Sixth French Symposium of Sedimentology, Montpellier, 17-18-19 Novembre 1997, Paris Publi ASF 27. pp. 105-106.

Ehrhold, A., 1999. Dynamique de comblement d'un bassin sédimentaire soumis à un régime mégatidal : exemple de la baie du Mont-SaintMichel. université de Caen Thèse de doctorat.

Gadd, P.E., Lavelle, W., Swift, D.J.P., 1978. Estimates of sand transport on the New York shelf using near-bottom current meter observations. J. Sed. Petrol. 48 (n¹), 239-252.

Grochowski, N.T.L., Collins, M.B., Boxall, S.R., Salomon, J.C., 1993. Sediment transport predictions for the English Channel, using numerical models. J. Geol. Soc. Lond. 150, 683-695.

Guillou, S., Nguyen, K.D., Hanich, L., Louaked, M., 1997. Modélisation numérique $2-\mathrm{D}$ de la circulation et de la pollution bactériologique dans la zone littorale de Granville. Rapport pour l'Agence de l'Eau de Basse Normandie, Vol. I et II. Lab de Mécanique université Caen.

Heathershaw, A.D., 1981. Comparisons of measured and predicted sediment transport rates in tidal currents. Mar. Geol. 42, 75-104.

Kenyon, N.H., 1970. Sand ribbons of european tidal seas. Mar. Geol. 9, 25-39.

Langhorne, D.N., 1981. An evaluation of Bagnold's dimensionless coefficient of proportionality using measurements of sand wave movement. Mar. Geol. 43, 49-64.

Larsonneur, C., 1977. La cartographie des dépôts meubles sur le plateau continental français : méthode mise au point et utilisée en Manche. J. Rech. Océanogr. vol. II (n²), 33-39.
Larsonneur, C., Walker, P., 1986. La dynamique sédimentaire dans le golfe normand-breton à l'aide des radionucléides artificiels et d'enregistrements au sonar latéral. Rapport CNEXO-université Caen.

Larsonneur, C., 1989. La Baie du Mont-Saint-Michel, un modèle de sédimentation en zone tempérée. Bull. Inst. Géol. Bas. Aquit. 46, 5-74.

Lees, B.J., 1983. The relationship of sediment transport rates and paths to sandbanks in a tidally dominated area off the coast of east Anglia U.K. Sedimentology 30, 461-483.

Levoy, F., 1994. Evolution et fonctionnement hydrosédimentaire des plages macrotidales : l'exemple de la côte Ouest du Cotentin. université de Caen Thèse de doctorat.

L'Homer, A., 1988. Les récifs à annélides (Hermelles) en baie du MontSaint-Michel - Écologie, géomorphologie, sédimentation et implications géologiques.

McLean, S.R., 1981. The role of non-uniform roughness in the formation of sand ribbons. Mar. Geol. 42, 49-74.

Migniot, C., 1992. Les lois de l'hydraulique appliquées à la sédimentologie dynamique. Revue Géologues 97, 41-48.

Nikodic, J., 1981. Dynamique sédimentaire dans la partie occidentale de la baie du Mont-Saint-Michel. université de Nantes Thèse de doctorat.

Nguyen, K.D., Ouahsine, A., 1997. 2D Numerical Study on Tidal Circulation in Strait of Dover. J. Water. Port Coastal and Ocean Engineering ASCE $123\left(n^{\circ} 1\right), 8-15$.

Nguyen, K.D., Martin, J.M., 1988. A two-dimensional fourth-order simulation for scalar transport in estuaries and coastal seas. Est. Coast. Shelf. Sci. 27 (3), 263-281.

Orbi, A., 1986. Circulation de marée dans le golfe normand-breton. université de Bretagne Occidentale Thèse de doctorat.

Seaward-Thomson, B.L., Hails, J.R., 1973. An appraisal of the computation of statistical parameters in grain size analysis. Sedimentology 20, 161-169.

Soulsby, R.L., 1983. The bottom boundary layer of shelf seas. In: Johns, B. (Ed.), Physical oceanography of coastal and shelf seas, 35, pp. 189-266.

Sternberg, R.W., 1972. Predicting initial motion of bedload transport sediment particles in the shallow marine environment. In: Swift, D.J.P., Duane, D.B., Pilkey, O.H. (Eds.), Shelf sediment transport, pp. 61-82.

Thauront, F., 1994. Les transits sédimentaires subtidaux dans les passes internes du bassin d'Arcachon. université de Bordeaux I Thèse de doctorat.

Walker, P., 2001. Dynamique sédimentaire dans le Golfe Norman-Breton : Intérêt de l'imagerie par sonar à balayage latéral. université de Caen Thèse de doctorat. 